\title{
Room temperature stable carbetocin for the prevention of postpartum haemorrhage during the third stage of labour in women delivering vaginally: study protocol for a randomized controlled trial
}

Mariana Widmer ${ }^{1 *}$, Gilda Piaggio², Hany Abdel-Aleem³ ${ }^{3}$ Guillermo Carroli ${ }^{4}$, Yap-Seng Chong ${ }^{5}$, Arri Coomarasamy ${ }^{6}$, Bukola Fawole ${ }^{7}$, Shivaprasad Goudar ${ }^{8}$, G. Justus Hofmeyr ${ }^{9}$, Pisake Lumbiganon ${ }^{10}$, Kidza Mugerwa ${ }^{11}$,

Thi My Huong Nguyen ${ }^{1}$, Zahida Qureshi ${ }^{12}$, Joao Paulo Souza ${ }^{13}$ and A. Metin Gülmezoglu ${ }^{1}$

\begin{abstract}
Background: Postpartum haemorrhage (PPH) is the leading cause of maternal mortality in low-income countries and contributes to nearly a quarter of maternal deaths globally. The current available interventions for prevention of postpartum haemorrhage, oxytocin and carbetocin, are limited by their need for refrigeration to maintain potency, as the ability to maintain a cold chain across the drug distribution and storage network is inconsistent, thus restricting their use in countries with the highest burden of maternal mortality. We describe a randomized, double-blind non-inferiority trial comparing a newly developed room temperature stable formulation of carbetocin to the standard intervention (oxytocin) for the prevention of PPH after vaginal birth.

Methods/design: Approximately 30,000 women delivering vaginally will be recruited across 22 centres in 10 countries. The primary objectives are to evaluate the non-inferiority of room temperature stable carbetocin (100 $\mu \mathrm{g}$ intramuscular) versus oxytocin (10 IU intramuscular) in the prevention of PPH and severe PPH after vaginal birth. The primary endpoints are blood loss $\geq 500 \mathrm{~mL}$ or the use of additional uterotonics (composite endpoint required by drug regulatory authorities) and blood loss $\geq 1,000 \mathrm{~mL}$ (WHO requirement). Non-inferiority will be assessed using a two-sided $95 \%$ confidence interval for the relative risk of the above endpoints for room temperature stable carbetocin versus oxytocin. The upper limit of the two-sided $95 \%$ confidence interval for the relative risk for the composite endpoint of blood loss $\geq 500 \mathrm{~mL}$ or the use of additional uterotonics, and for the endpoint of blood loss $\geq 1,000 \mathrm{~mL}$, will be compared to a non-inferiority margin of 1.16 and 1.23 , respectively. If the upper limit is below the corresponding margin, non-inferiority will have been demonstrated. The safety analysis will include all women receiving treatment. Safety and tolerability will be assessed by a review of adverse events, by conducting inferential testing with significance levels for between-group comparisons.

(Continued on next page)
\end{abstract}

\footnotetext{
* Correspondence: widmerm@who.int

'Department of Reproductive Health and Research, World Health

Organization, UNDP/UNFPA/UNICEF/WHO/World Bank Special Programme of

Research, Development and Research Training in Human Reproduction

(HRP), Avenue Appia 20, Geneva 1201, Switzerland

Full list of author information is available at the end of the article
} 
(Continued from previous page)

Discussion: If the results of the study show that room temperature stable carbetocin is a safe and effective alternative to oxytocin, this could have a substantial impact on the prevention of postpartum haemorrhage and maternal survival worldwide.

Trial registration: ACTRN12614000870651 (14 August 2014)

Keywords: Postpartum haemorrhage, Room temperature stable carbetocin, Oxytocin, Non-inferiority trial

\section{Background}

Postpartum haemorrhage (PPH) is defined as a blood loss of $\geq 500 \mathrm{~mL}$ within 24 hours of delivery, while severe $\mathrm{PPH}$ ( $\mathrm{sPPH}$ ) is defined as a blood loss of $\geq 1,000 \mathrm{~mL}$ within the same time frame [1]. PPH is the leading cause of maternal mortality in low-income countries, contributing to nearly a quarter of maternal deaths globally. In addition, $\mathrm{PPH}$ is a significant contributor to severe maternal morbidity and long-term disability, as well as to a number of other severe maternal conditions generally associated with more substantial blood loss, including shock and organ dysfunction [2-4]. Improving health care for women during childbirth in order to prevent and treat PPH is an essential step towards the achievement of the United Nations Millennium Development Goals.

The majority of deaths due to PPH could be avoided through the use of prophylactic uterotonics during the third stage of labour and by timely and appropriate management. Injectable oxytocin has been recommended by the World Health Organization (WHO) for routine use during the third stage of labour and is the preferred drug for the prevention and management of blood loss after childbirth; however, several studies have demonstrated that oxytocin loses potency in field conditions, particularly in tropical climates $[1,5,6]$. To decrease potency loss due to degradation, oxytocin must be either stored at controlled room temperature $\left(25^{\circ} \mathrm{C}\right.$ or lower) for a restricted amount of time or refrigerated $\left(2{ }^{\circ} \mathrm{C}\right.$ to $\left.8{ }^{\circ} \mathrm{C}\right)$, making its use difficult in low resource settings. To ease this barrier, several groups have been researching heat stable oxytocin formulations [5]. Though some progress has been made in this area, there is currently no heat stable oxytocin formulation for therapeutic use [7].

Carbetocin (1-deamino-1-monocarba-(2-O-methyltyrosine)-oxytocin) is a long-acting synthetic agonist analogue of the human oxytocin, and it appears to be a promising agent in the prevention of $\mathrm{PPH}$ following vaginal delivery. The clinical and pharmacological properties of carbetocin are similar to those of oxytocin. However, carbetocin is a more stable molecule and induces a prolonged uterine response, when administered postpartum, in terms of both amplitude and frequency of contractions due to its longer half-life [8-10]. In a Cochrane systematic review including 11 studies (2,635 women), the use of carbetocin resulted in a statistically significant reduction in the use of additional/therapeutic uterotonics compared to oxytocin for those who underwent caesarean delivery (risk ratio (RR) 0.62; $95 \%$ confidence interval (CI) 0.44 to 0.88 ; four trials, 1,173 women), but not for vaginal delivery. Compared to oxytocin, carbetocin was associated with reduced uterine massage following both caesarean delivery (RR 0.54; $95 \%$ CI 0.37 to 0.79; two trials, 739 women) and vaginal delivery (RR 0.70; $95 \%$ CI 0.51 to 0.94; one trial, 160 women). There were no statistically significant differences between carbetocin and oxytocin in terms of risk of any PPH or in risk of sPPH [11].

Carbetocin is currently approved in multiple countries for the prevention of $\mathrm{PPH}$ during caesarean section. The drug is licensed to be administered by slow intravenous (IV) single injection at a dose of $100 \mu \mathrm{g}$. Its current formulation requires refrigeration.

A room temperature stable (RTS) variant of carbetocin has recently been developed and is now approved in the European Union; this variant differs from the current carbetocin formulation only in its excipients (Table 1). The stability data from long-term studies performed at $30{ }^{\circ} \mathrm{C}$ and $75 \%$ relative humidity, as well as accelerated at $40{ }^{\circ} \mathrm{C}$ and $75 \%$ relative humidity, indicate a shelf life of at least 36 months at $30{ }^{\circ} \mathrm{C}$ for the new RTS formulation of carbetocin. Carbetocin RTS therefore represents a promising intervention for reducing $\mathrm{PPH}$, particularly in settings where cold storage is difficult to achieve and maintain. Here, we describe a planned randomized trial to evaluate the non-inferiority of carbetocin RTS versus oxytocin after vaginal delivery in the prevention of $\mathrm{PPH}$ and $\mathrm{sPPH}$.

\section{Methods/design}

\section{Primary objectives and hypotheses}

The trial has two primary objectives: 1) To evaluate non-inferiority of carbetocin RTS versus oxytocin after vaginal delivery in the prevention of the composite endpoint "blood loss of $500 \mathrm{~mL}$ or more or the use of additional uterotonics" at one hour and up to two hours for women who continue to bleed after one hour; and 2) to evaluate non-inferiority of carbetocin RTS versus oxytocin in the prevention of $\mathrm{sPPH}(\geq 1,000 \mathrm{~mL}$ blood loss $)$ at one hour and up to two hours for women who continue to bleed after one hour. 
Table 1 Composition of carbetocin RTS and carbetocin

\begin{tabular}{|c|c|c|c|c|c|}
\hline \multicolumn{3}{|c|}{ New carbetocin RTS formulation } & \multicolumn{3}{|c|}{ 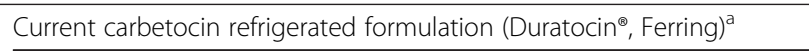 } \\
\hline Component & Amount $(\mathrm{mg} / \mathrm{mL})$ & Function & Component & Amount $(\mathrm{mg} / \mathrm{mL}$ & Function \\
\hline Carbetocin & 0.100 & Active substance & Carbetocin & 0.100 & Active substance \\
\hline Succinic acid & 1.19 & Buffer & Sodium chloride & 9 & Isotonicity agent \\
\hline Mannitol & 47.0 & Isotonicity agent & Glacial acetic acid & to $\mathrm{pH} 3.8$ & pH adjustment \\
\hline L-methionine & 1.00 & Antioxidant & Water for injection & $1.0 \mathrm{~mL}$ & Solvent \\
\hline Sodium hydroxide $2 \mathrm{~N}$ & to $\mathrm{pH} 5.45$ & $\mathrm{pH}$ adjustment & - & - & - \\
\hline Water for injection & $1.0 \mathrm{~mL}$ & Solvent & - & - & - \\
\hline
\end{tabular}

${ }^{a}$ Carbetocin $100 \mu \mathrm{g} / \mathrm{mL}$ was first approved in Canada in June 1997 and is currently registered in more than 70 countries under the trade names PABAL/DURATOCIN/LONACTENE/DURATOBAL

RTS room temperature stable

For both objectives the hypotheses are: 1) carbetocin RTS is non-inferior to oxytocin in terms of the proportion of women with blood loss $\geq 500 \mathrm{~mL}$ or the use of additional uterotonic drugs after vaginal delivery within a non-inferiority margin of 1.16 on the relative risk scale, and the proportion of women with blood loss $\geq 1,000 \mathrm{~mL}$ after vaginal delivery within a non-inferiority margin of 1.23 on the relative risk scale; and 2) carbetocin RTS is superior to oxytocin in terms of the proportion of women with blood loss $\geq 500 \mathrm{~mL}$ or use of additional uterotonic drugs, and in terms of the proportion of women with blood loss $\geq 1,000 \mathrm{~mL}$ after vaginal delivery. For each of the two primary endpoints, superiority will be tested if non-inferiority has been demonstrated.

Investigators and other assessors will be blinded to individual treatment allocation. The study will be conducted by the HRP/WHO, and WHO staff will also remain blinded to individual participant treatment allocation during the conduct of the trial.

\section{Study drug}

Carbetocin RTS (Ferring, St. Prex, Switzerland) will be administered as a single IM dose of $100 \mu \mathrm{g}$ in a $1 \mathrm{~mL}$ solution. Oxytocin $10 \mathrm{IU} / \mathrm{mL}$ (Syntocinon ${ }^{\circ}$, Novartis, Basel, Switzerland) will be administered as a single intramuscular (IM) dose in a $1 \mathrm{~mL}$ solution. In order to maintain the blindness of the trial, carbetocin RTS and oxytocin will be provided in $1 \mathrm{~mL}$ ampoules in consecutively numbered treatment packs arranged in dispensers and will be stored in a refrigerator at $2{ }^{\circ} \mathrm{C}$ to $8{ }^{\circ} \mathrm{C}$.

\section{Study design}

This clinical trial will be conducted in compliance with the clinical trial protocol, good clinical practice and the applicable regulatory requirements. The trial is planned to be conducted at 22 centres in 10 countries: Argentina, Egypt, India, Kenya, Nigeria, Singapore, South Africa, Thailand, Uganda and the United Kingdom. Approximately 30,000 women will be included in the trial. The recruitment period will be approximately 12 months in each country.
The care provider in charge of antenatal care visits at the hospital will inform potentially eligible pregnant women (women with no allergies to carbetocin, other oxytocin homologues or excipients, or serious cardiovascular disorders) about the trial.

At admission for labour at the hospital, the woman will be approached for participation in the trial when she is in early labour and if her vital signs are normal, and she is not stressed. The investigator will invite her to sign the informed consent. Informed consent will be obtained before any trial-related procedures are carried out.

During the second stage of labour when vaginal delivery is imminent, the woman will be randomized to receive either a single dose of oxytocin 10 IU IM or a single dose of carbetocin RTS $100 \mu \mathrm{g}$ IM.

The investigator will administer the investigational medicinal product (IMP) immediately after the birth of the baby (preferably within one minute). Once the IMP has been administered, the investigator will follow the management of the third stage of labour as recommended in WHO guidelines and as detailed in the trial's manual of operations.

Once the cord is clamped (1-3 minutes after delivery of the baby) and cut, the investigator will place a calibrated drape under the woman's buttocks and blood loss will be measured for one hour or two hours postpartum if the bleeding continues beyond one hour (blood loss measuring details are provided in the trial's manual of operations).

The woman will end her participation in the trial after her discharge or if she is transferred to a higher care unit, such as an intensive care unit (ICU). Data will be entered into a validated online database system compliant with the FDA 21 CFR part 11.

The trial master protocol was approved by the World Health Organization Research Ethics Review Committee. All sites received ethics approval from their institutional/national boards (see additional file 1 for the list of ethics committees that reviewed and approved the proposal). 


\section{Inclusion/exclusion criteria}

Women will be eligible for inclusion into this trial if they are expected to deliver vaginally, have a cervical dilatation equal to or less than $6 \mathrm{~cm}$, have a known singleton pregnancy and have provide written informed consent. Women will not be eligible for study participation if they: 1) are in an advanced first stage of labour $(>6 \mathrm{~cm}$ cervical dilatation) or are too distressed to understand, confirm and give informed consent regardless of cervical dilatation; 2) are non-emancipated minors (as per local regulations) without a guardian; 3) have been scheduled for a planned caesarean section; 4) have a birth considered to be an abortion according to local guidelines; 5 ) have known allergies to carbetocin, other oxytocin homologues or excipients in the medicinal products used in the trial; 6) have serious cardiovascular disorders; 7) have serious hepatic or renal disease; 8) have epilepsy; or 9) are not capable of giving consent due to other health problems such as obstetric emergencies (for example, antepartum haemorrhage) or mental disorder.

Participants will be free to discontinue the trial at any time without giving their reason(s). Participants who withdraw will not be replaced; that is, randomized numbers will be uniquely linked to each participant. Data collected up to that point will be included unless participants request the opposite. After a participant has completed the trial or has withdrawn early, usual treatment will be administered, if required, in accordance with the trial centre's standard of care and generally accepted medical practice and depending on the participant's individual medical needs.

\section{Randomization}

The random allocation sequence will be generated centrally at WHO headquarters using computer-generated random numbers. Randomization will be stratified by country and will use permuted blocks, with an allocation ratio of 1:1. Participants will randomly be assigned within each country to one of the two treatment groups: oxytocin 10 IU IM or carbetocin RTS $100 \mu \mathrm{g}$ IM. Allocation of the randomly generated sequence will be performed by consecutively numbered treatment packs arranged in dispensers.

\section{Primary and secondary endpoints}

The primary and secondary endpoints are listed in Table 2. The trial is being conducted as an effectiveness trial with the objective of carbetocin RTS being registered for the indication "prevention of postpartum haemorrhage" by drug regulatory authorities if it is shown to be non-inferior or superior to oxytocin. The primary composite endpoint is blood loss $\geq 500 \mathrm{~mL}$ or use of additional uterotonics. For the purpose of evaluating clinical effectiveness and for the potential inclusion
Table 2 Primary and secondary endpoints

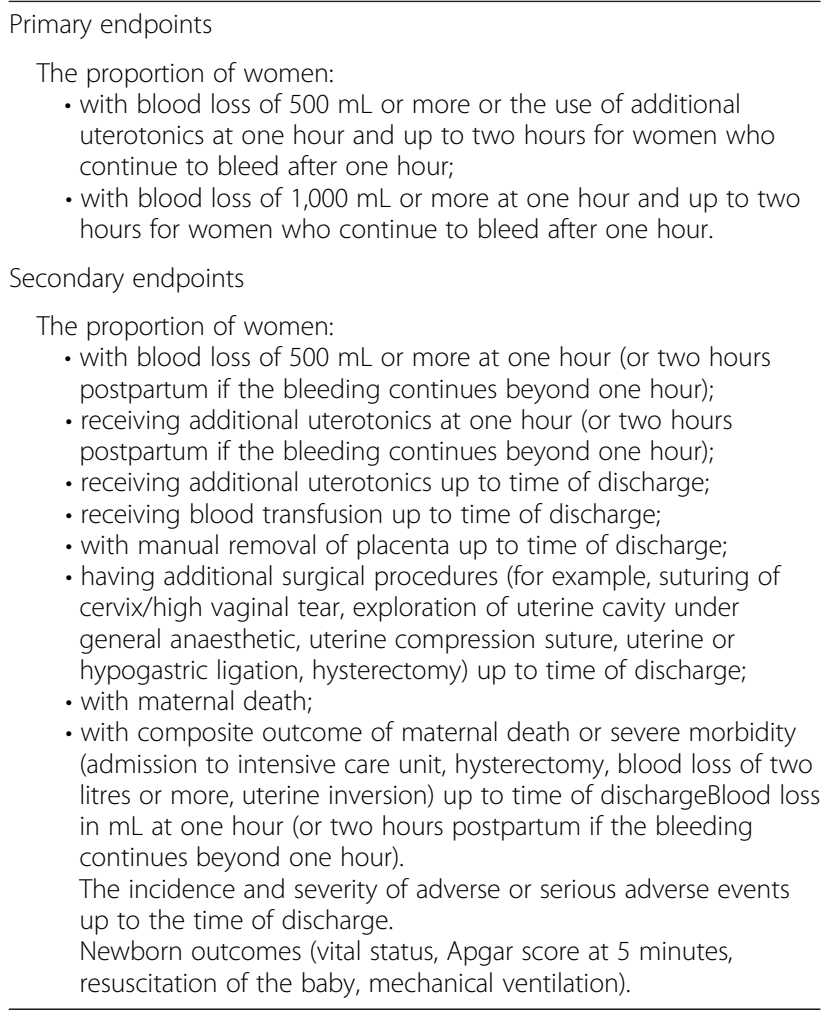

of carbetocin RTS in future WHO guidelines and the Model List of Essential Medicines, the more substantive endpoint of blood loss $\geq 1,000 \mathrm{~mL}$ is also a primary endpoint. Since the two primary endpoints serve independent objectives, there will be no adjustment for the type I error rate for multiplicity of endpoints.

\section{Assessment of efficacy}

Efficacy will be assessed using blood loss volume measurement per the two primary endpoints listed in Table 2. Blood loss will be measured using a calibrated drape [12], which will be placed under the participant's buttocks once the cord is clamped (1-3 minutes after delivery of the baby) and cut. Blood loss will be measured for one hour, or for two hours postpartum, if the bleeding continues beyond one hour. Blood loss weight in grams will be converted to millilitres by dividing the figure in grams by 1.06 (blood density in grams per millilitre) [13].

\section{Assessment of safety}

Comprehensive assessment of the safety of the study drugs will be performed throughout the course of the trial, from the time of the participant's signature of informed consent until discharge. Trial centre personnel will report any adverse event (AE), whether observed by the investigator or reported by the participant. 
The intensity of an AE will be classified using a threepoint scale: mild - awareness of signs or symptoms, but no disruption of usual activity; moderate - event sufficient to affect usual activity; and severe - inability to work or perform usual activities. AEs will also be classified as possibly related to study drug (that is, there is evidence or argument to suggest a causal relationship between the $\mathrm{AE}$ and the study drug), or not related to study drug (that is, there is no reasonable evidence or argument to suggest a causal relationship between the $\mathrm{AE}$ and the study drug).

The outcome of an AE will be classified as: recovered/ resolved (fully recovered or the condition has returned to the level observed at initiation of trial treatment); recovered/resolved with sequelae (resulted in persistent or significant disability/incapacity); recovering/resolving; not recovered/not resolved; or fatal.

An AE will be considered serious if it results in death, is life-threatening, requires in-patient hospitalization or prolongation of existing hospitalization, results in persistent or significant disability/incapacity, is a congenital anomaly/birth defect, or is an important medical event. If an investigator becomes aware of a serious $\mathrm{AE}$ after the participant's hospital discharge, and he/she assesses the serious AE to have a reasonable possible causality to the study drug, the case will be reported, regardless of how long after the end of the trial the AE took place.

Since the amount of blood lost is collected as an efficacy endpoint, only the following blood loss events that fulfil the criteria for a serious AE will be recorded: ICU admission (adult ICU admission, not maternity/labour ward high care); surgical intervention for control of haemorrhage (hypogastric artery ligation or uterine compression sutures); peripartum hysterectomy, blood loss $\geq 2,000 \mathrm{~mL}$; and uterine inversion.

\section{Sample size calculation}

The two endpoints that guided the sample size estimation for the trial were sPPH (blood loss $\geq 1,000 \mathrm{~mL}$ ), $\mathrm{PPH}$ (blood loss $\geq 500 \mathrm{~mL}$ ) or the use of additional uterotonics. Severe PPH is a more serious endpoint that is closely associated to severe maternal morbidity and related to additional interventions. The sPPH endpoint is also less frequent and thus guided the overall sample size estimation.

A non-inferiority design was chosen since the aim of the trial is to determine if carbetocin RTS is non-inferior in efficacy to the standard intervention (oxytocin $10 \mathrm{IU}$ IM). In order to demonstrate non-inferiority within a margin of $0.46 \%$, with a power of $80 \%$ and with a significance level of $2.5 \%$, a total of 29,082 women are needed assuming an equal sPPH prevalence of $2 \%$ with both treatments, calculated by the standard formula (see Additional file 2 for the rationale of the choice of the margin) [14]. The margin of $0.46 \%$ on the difference scale corresponds to 1.23 on the relative scale for the assumed prevalence. Figure 1 shows the total sample size required to assess non-inferiority at the $2.5 \%$ level of significance (usually required for non-inferiority trials) for different scenarios. We also assumed, from a similar large trial [15], a $3 \%$ loss of study participants who are eligible to contribute to the analysis due to exclusion of women

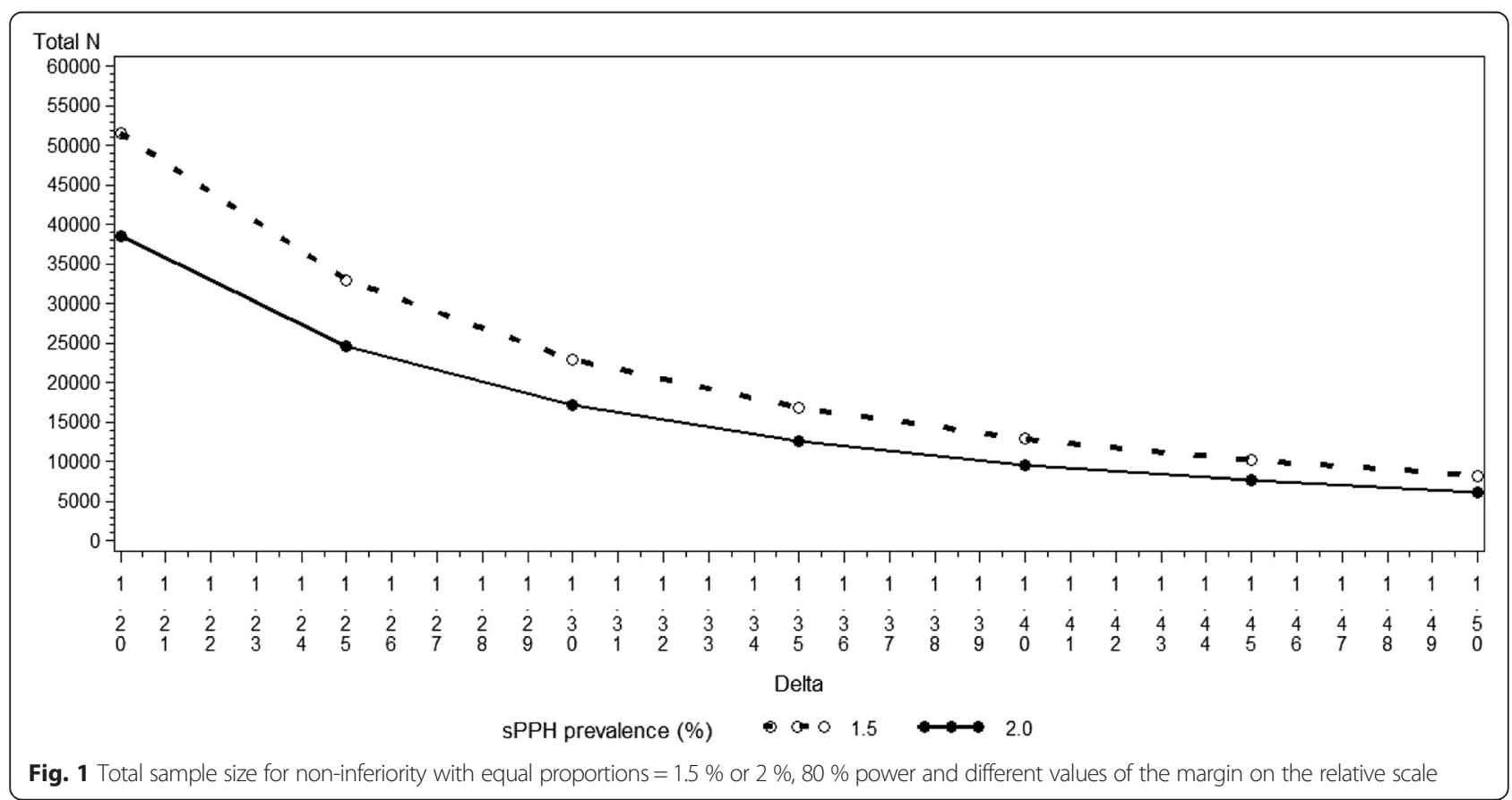


with a caesarean section or abortion after randomization, and those who are not protocol-compliant (excluded only in the per-protocol analysis), bringing the sample size to 30,000. This sample size will provide $90 \%$ power for a conventional two-sided $5 \%$ test of superiority to detect a minimum significant difference between $1.5 \%$ and $2 \%$ in the rate of sPPH for the two treatment groups.

Similarly, we calculated the sample size for the composite endpoint defined as blood loss $\geq 500 \mathrm{~mL}$ or administration of additional uterotonics. With active management, the risk of this event is estimated as $16 \%$ (personal communication) [15]. Assuming equal prevalence for this event of $16 \%$ with both treatments, in order to demonstrate non-inferiority within a margin of 1.16 on the relative scale, with a power of $80 \%$ and with a significance level of $2.5 \%$, a total of 6,242 women are needed. With 30,000 women the power obtained would be more than $99 \%$ [14]. This sample size will provide a power of $99.5 \%$ for a conventional two-sided $5 \%$ test of superiority to detect a minimum significant difference between $16 \%$ and $18 \%$ in the occurrence of $\mathrm{PPH}$ or administration of additional uterotonics for the two treatment groups (Stata version 13.1).

\section{Analysis populations}

Analyses will be performed in three populations: intention-to-treat (ITT), modified ITT, and per-protocol. The ITT population includes all randomized participants, using the treatment as randomized. The modified ITT analysis excludes women having a caesarean section after randomization and those withdrawing consent. The per-protocol population includes all participants randomized into the trial, who were compliant with, and treated according to, the protocol, and who fulfil the following criteria: compliance with all entry criteria, absence of major clinical trial protocol violations with respect to factors likely to affect the efficacy of treatment, and adequate compliance with trial medication (giving oxytocin or carbetocin to women as randomized). Declaring carbetocin RTS non-inferior to oxytocin will require non-inferiority to be demonstrated for both per-protocol and the modified ITT analyses. For testing the superiority of each endpoint, the modified ITT analysis will be the main analysis. The primary population for safety analysis will consist of all women receiving treatment.

\section{Statistics}

Safety and tolerability will be assessed by a review of AEs, by conducting inferential testing with significance levels for between-group comparisons. The betweentreatment difference for the occurrence of AEs will be tested using the stratified Mantel-Haenszel chi-square if frequencies are sufficiently high, or otherwise with exact methods taking stratification into account.

For efficacy, non-inferiority will be assessed using a two-sided $95 \%$ confidence interval $(\mathrm{CI})$ for the relative risk (RR) of sPPH and of $\mathrm{PPH}$ or additional uterotonic use (carbetocin RTS versus oxytocin). The upper limit of the two-sided $95 \% \mathrm{CI}$ for the RR for the composite endpoint of blood loss $\geq 500 \mathrm{~mL}$ or additional uterotonic use will be compared to the non-inferiority margin of 1.16 . The upper limit of the two-sided $95 \%$ CI for the RR for the endpoint of blood loss $\geq 1,000 \mathrm{~mL}$ will be compared to the non-inferiority margin of 1.23 . If the upper limit is below the corresponding margin, non-inferiority will have been demonstrated. This upper limit is the same as the upper limit of the one-sided $97.5 \% \mathrm{CI}$; therefore, the significance level for the non-inferiority test will be $2.5 \%$ (one-sided). For each endpoint, to declare carbetocin RTS non-inferior to oxytocin, we will require noninferiority to be demonstrated for both PP and the modified ITT analyses. If non-inferiority is demonstrated, a two-tailed superiority test will be conducted at $5 \%$ level of significance. Interpretation will be as in Fig. 1 of Piaggio et al. [16]. Comparisons will also be expressed as risk differences with $95 \%$ CIs.

The statistical technique used to conduct tests and obtain CIs for the main endpoints will be a logistic model with a binary endpoint, a binomial distribution and the log link to obtain RRs. The identity link will be used to obtain risk differences. Stratifying variables (country) will be included in the model. The model will be fitted using SAS Software version 9.3 (SAS Institute Inc., Cary, NC, USA). A separate model will be fitted for each of the two primary endpoints.

We will use the RR as measure of treatment effect on the relative scale for the two primary endpoints. For the composite endpoint of blood loss $\geq 500 \mathrm{~mL}$ or use of additional uterotonics, the margin has been justified as 1.2 on the odds ratio (OR) scale and 1.16 on the RR scale. Heterogeneity across centres will be assessed by using a term in the logistic model for the interaction between treatment and centres. If there is heterogeneity between the centres for any of the results, the possible causes will be explored.

The secondary binary endpoints of blood loss $\geq 500 \mathrm{~mL}$, use of additional uterotonics, blood transfusion, manual removal of placenta, additional surgical procedures, maternal death and the composite endpoint of maternal death or severe morbidity, will be analysed using the modified ITT population and will be assessed only for conventional superiority using risk differences and relative risks with $95 \%$ CIs estimated with the same techniques described for the main endpoints.

The secondary outcome of blood loss in millilitres will be analysed using the log transformation. This is based on the following: 1) the blood loss distribution is 
positively skewed; 2) different distributions were fitted to blood loss data from a large trial [15] and the lognormal distribution was found to have a very good fit (personal communication); and 3) the lognormal distribution was used for blood loss data in the literature [13]. Therefore, a lognormal distribution will be fitted to the blood loss data and the probabilities of blood loss $\geq 500 \mathrm{~mL}$ and of $\geq 1,000 \mathrm{~mL}$ will be compared between treatments using parametric methods. The quantiles will be compared between treatments using quantile regression [17].

\section{Trial oversight}

The trial will be overseen by two committees. The Trial Steering Committee (TSC) is composed of the WHO/ HRP coordination team, investigators from the 10 participating countries and five independent members with expertise in clinical, statistical and scientific disciplines. The TSC should provide oversight of the progress of the trial and ensure the trial is conducted in accordance with the principles of good clinical practice (GCP).

The Data and Safety Monitoring Committee (DSMC), with no direct involvement in the trial, will handle any ethical issues that may arise while the trial is in progress and will scrutinize two interim analyses, the first to look at safety when 5,000 participants have been recruited, and the second conducted when 15,000 women have been recruited to look at both safety and efficacy. The interim analyses will be masked to trial investigators, trial statistician, WHO, Merck for Mothers and Ferring staff, but not to DSMC members. The DSMC will be expected to provide an ongoing risk-benefit evaluation that addresses the uncertainty necessary to continue.

\section{Discussion}

$\mathrm{PPH}$ is one of the major contributors to maternal mortality and morbidity worldwide. Prevention of PPH is therefore of great importance in the pursuit of improved health care for women. The decision to proceed with a large, randomized controlled trial to address this unmet medical need by evaluating the effectiveness of carbetocin RTS compared to oxytocin was based on several considerations. This included the frequent concerns about the quality of oxytocin and its stability in some developing countries, the potential advantage of carbetocin RTS due to its longer half-life (than that of oxytocin), especially when administered intramuscularly, and its heat stability. This study will be conducted under conditions where both study drugs, oxytocin and carbetocin RTS, will be kept under strict refrigeration. If the trial meets its primary endpoint of non-inferiority to oxytocin, the carbetocin RTS formulation will be made available in high-burden countries at an accessible public sector price. This would have major implications for expanding access to effective care and could have a substantial impact on maternal survival.

The risk-benefit relationship was carefully considered in the planning of the trial, as was the selection of the dose of carbetocin RTS. The selected dose for the carbetocin RTS IM injection is $100 \mu \mathrm{g}$, the same as the dose approved for IV injection following caesarean section. The safety profile of a $100 \mu \mathrm{g}$ dose of the carbetocin refrigerated formulation, when administered intravenously, is derived from clinical studies as well as from pharmacovigilance activities that comprise nearly 5 million women exposed. Reported AEs during clinical trials when administered by IV after caesarean section under spinal or epidural anaesthesia are headache, tremor, hypotension, flushing, nausea, abdominal pain, pruritus, anaemia, dizziness, chest pain, dyspnoea, metallic taste and vomiting. The AEs observed were of the same type and frequency as those observed with the comparator, oxytocin. In addition, efficacy and safety data on $100 \mu \mathrm{g}$ of the carbetocin refrigerated formulation delivered intramuscularly during vaginal delivery is available from several published randomized clinical trials [11, 18, 19]. Although the population from whom these safety data are generated is different from the study population of this protocol, women undergoing caesarean section are expected to be at the same or higher risk of AEs than women having a vaginal delivery. It is therefore expected that a carbetocin RTS dose of $100 \mu \mathrm{g}$ IM, when used in women after vaginal delivery, will be efficacious and have a safety profile that is equal to or better than the well-characterized safety profile of $100 \mu \mathrm{g}$ IV when used in women following caesarean section.

The study has several strengths. It is a global, multicentred, randomized, double-blind, active comparator controlled study. The active comparator is oxytocin, which is the most commonly recommended drug for PPH prophylaxis and considered the current gold standard in WHO guidelines. The $10 \mathrm{IU}$ dose of oxytocin was chosen as this is currently the most commonly used dose in clinical practice internationally when IM administration is practiced. Blood loss of $\geq 1,000 \mathrm{~mL}$ is a primary endpoint in this trial, as it was considered as one of the three critical endpoints (together with blood transfusion and maternal death) for the earlier 2007 WHO recommendations for PPH prevention where outcomes were rated by an independent panel [1]. We also used reliable estimates of prevalence of blood loss $\geq 1,000 \mathrm{~mL}$ under active management with oxytocin from different trials and systematic reviews. Additionally, an external DSMC has been established for the ongoing assessment of the risk-benefit ratio. One limitation of our study is that the predicted sample size makes assumptions that in reality might not hold. For example, if the efficacy of oxytocin is much better than that of carbetocin RTS, this would imply that non-inferiority is not likely to be demonstrated and the trial should be stopped for futility. If 
oxytocin is just slightly better than carbetocin RTS, the predicted sample size could have been underestimated, but this is not likely given previous evidence [11].

Results of this trial will be extremely useful, particularly in tropical settings, where cold storage is difficult to achieve and maintain. Should this trial demonstrate that carbetocin RTS is non-inferior to oxytocin in preventing postpartum haemorrhage, in settings where the cold chain cannot be guaranteed, oxytocin could be replaced by carbetocin RTS as the uterotonic used during the third stage of labour. In addition, and based on the tripartite agreement signed between WHO/HRP, Merck for Mothers and Ferring Pharmaceuticals, carbetocin RTS could be available in high-burden countries at a public sector price comparable to oxytocin's price. This could have major implications for preventing $\mathrm{PPH}$ and a substantial impact on maternal survival worldwide.

\section{Trial status}

The trial has started recruitment in five countries. The other five countries are expected to receive regulatory authorities' approval before April 2016.

\section{Additional files}

Additional file 1: Countries ethics committees list and status of the project approval request. (DOCX $15 \mathrm{~kb}$ )

Additional file 2: Rationale for the choice of the non-inferiority trial the choice of the non-inferiority trial [20-22]. (DOCX $24 \mathrm{~kb}$ )

\section{Abbreviations}

AE: adverse event; Cl: confidence interval; DSMC: Data Safety Monitoring Committee; GCP: good clinical practice; HRP: the UNDP/UNFPA/UNICEF/ WHO/World Bank Special Programme of Research, Development and Research Training in Human Reproduction; IM: intramuscular; IMP: investigational medicinal product; ITT: intention to treat; IU: international unit; IV: intravenous; $\mathrm{mL}$ : millilitres; PPH: postpartum haemorrhage; RHR: reproductive health and research; RR: risk ratio; RTS: room temperature stable; SAE: serious adverse event; sPPH: severe postpartum haemorrhage: TSC: Trial Steering Committee; WHO: World Health Organization.

\section{Competing interests}

The authors declare that they have no competing interests. WHO/HRP is solely responsible for the trial. Merck for Mothers is providing the funding and Ferring Pharmaceuticals the carbetocin RTS. The collaboration between WHO/HRP, Merck for Mothers and Ferring Pharmaceuticals is governed by a tripartite agreement.

\section{Authors' contributions}

The protocol was written by AMG, GP and MW (WHO/HRP coordination team) with comments and contributions from HA, GC, YSC, AC, BF, SG, GJH, PL, KM, TMHN, ZQ and JPS (country investigators and members of the Trial Steering Committee). All authors contributed to the development of the trial protocol and approved the submitted manuscript.

\section{Acknowledgements}

We thank colleagues from Merck, Sharp \& Dohme Corp. and Ferring Pharmaceuticals for their comments and contributions to the protocol. We thank Novartis Pharma AG for donating the oxytocin and Ferring Pharmaceuticals for donating the carbetocin RTS.

\section{Funding}

The study to be undertaken pursuant to this protocol is funded by Merck Sharp \& Dohme Corp., a wholly owned subsidiary of Merck and Co., Inc. (Kenilworth, NJ USA), through its Merck for Mothers program. The authors are responsible for the design of the protocol and the planning, monitoring and implementation of the study.

\section{Author details}

${ }^{1}$ Department of Reproductive Health and Research, World Health Organization, UNDP/UNFPA/UNICEF/WHO/World Bank Special Programme of Research, Development and Research Training in Human Reproduction (HRP), Avenue Appia 20, Geneva 1201, Switzerland. ${ }^{2}$ Medical Statistics deparment, London School of Hygiene and Tropical Medicine, London, UK. ${ }^{3}$ Department of Obstetrics and Gynecology, Women's Health Hospital, Faculty of Medicine, Assiut University, Assiut, Egypt. ${ }^{4}$ Centro Rosarino de Estudios Perinatales, Rosario, Argentina. ${ }^{5}$ Department of Obstetrics and Gynaecology, Yong Loo Lin School of Medicine, National University Hospital Singapore, Singapore, Singapore. 'Birmingham Women's NHS Foundation Trust, Birmingham, UK. ${ }^{7}$ Department of Obstetrics and Gynaecology, College of Medicine, University of Ibadan, Ibadan, Nigeria. ${ }^{8}$ Department of Medical Education, K L E Society's J N Medical College, Belgaum, India. ${ }^{9}$ Effective Research Care Unit, University of Witwatersrand, University of Fort Hare, East London, South Africa. ${ }^{10}$ Department of Obstetrics and Gynaecology, Khon Kaen University, Khon Kaen, Thailand. ${ }^{11}$ Department of Obstetrics and Gynecology, School of Medicine, Makerere University College of Health Sciences, Kampala, Uganda. ${ }^{12}$ Department of Obstetrics and Gynaecology, Kenyatta National Hospital, University of Nairobi, Nairobi, Kenya.

${ }^{13}$ Department of Social Medicine, Ribeirão Preto Medical School, University of São Paulo, Ribeirão Preto, SP, Brazil.

Received: 16 June 2015 Accepted: 2 March 2016

Published online: 17 March 2016

\section{References}

1. WHO. Recommendations for the prevention and treatment of postpartum haemorrhage. Geneva, Switzerland: World Health Organization; 2012.

2. Khan KS, Wojdyla D, Say L, Gulmezoglu AM, Van Look PF. WHO analysis of causes of maternal death: a systematic review. Lancet. 2006;367(9516):1066-74.

3. Campbell OM, Graham WJ. Lancet Maternal Survival Series steering group. Strategies for reducing maternal mortality: getting on with what works. Lancet. 2006:368(9543):1284-99.

4. Souza JP, Gülmezoglu AM, Vogel J, Carroli G, Lumbiganon P, Qureshi Z, Costa MJ, Fawole B, Mugerwa Y, Nafiou I, Neves I, Wolomby-Molondo JJ, Bang HT, Cheang K, Chuyun K, Jayaratne K, Jayathilaka CA, Mazhar SB, Mori R, Mustafa ML, Pathak LR, Perera D, Rathavy T, Recidoro Z, Roy M, Ruyan P, Shrestha N, Taneepanichsku S, Tien NV, Ganchimeg T, Wehbe M, Yadamsuren B, Yan W, Yunis K, Bataglia V, Cecatti JG, Hernandez-Prado B, Nardin JM, Narváez A, Ortiz-Panozo E, Pérez-Cuevas R, Valladares E, Zavaleta $\mathrm{N}$, Armson A, Crowther C, Hogue C, Lindmark G, Mittal S, Pattinson R, Stanton ME, Campodonico L, Cuesta C, Giordano D, Intarut N, Laopaiboon M, Bahl R, Martines J, Mathai M, Merialdi M, Say L. Moving beyond essential interventions for reduction of maternal mortality (the WHO Multicountry Survey on Maternal and Newborn Health): a cross-sectional study. Lancet. 2013:9879(381):1747-55.

5. Heat-stable oxytocin - Technology opportunity assessment report. PATH, February 2012.

6. Hogerzeil HV, Walker GJA, de Goeje MJ. Stability of injectable oxytocics in tropical climates: results of field surveys and simulation studies on ergometrine, methylergometrine and oxytocin. 1993

7. Ashigbie P. Priority Medicines for Europe and the World 2013 Update. Background paper 6.16 - Postpartum haemorrhage. Geneva, Switzerland: WHO; 2013.

8. Ryden $\mathrm{G}$, Sjoholm I. Half-life of oxytocin in blood of pregnant and non-pregnant women. Acta Endocrinol (Copenh). 1969:61(3):425-31.

9. Fabian M, Forsling ML, Jones JJ, Pryor JS. The clearance and antidiuretic potency of neurohypophysial hormones in man, and their plasma binding and stability. J Physiol. 1969;204(3):653-68.

10. Chard T, Boyd NR, Forsling ML, MCNeilly AS, Landon J. The development of a radioimmunoassay for oxytocin: the extraction of oxytocin from plasma, and 
its measurement during parturition in human and goat blood. J Endocrinol. 1970;48(2):223-34.

11. Su LL, Chong YS, Samuel M. Carbetocin for preventing postpartum haemorrhage. Cochrane Database Syst Rev. 2012;4:CD005457.

12. Patel A, Goudar SS, Geller SE, et al. Drape estimation vs. visual assessment for estimating postpartum hemorrhage. Int J Gynaecol Obstet. 2006;93(3):220-4.

13. Althabe F, Aleman A, Tomasso G, et al. A pilot randomized controlled trial of controlled cord traction to reduce postpartum blood loss. Int I Gynaecol Obstet. 2009;107(1):4-7.

14. Jones B, Jarvis P, Lewis JA, Ebbutt AF. Trials to assess equivalence: the importance of rigorous methods. BMJ. 1996;313(7048):36-9.

15. Gulmezoglu AM, Lumbiganon P, Landoulsi S, et al. Active management of the third stage of labour with and without controlled cord traction: a randomised, controlled, non-inferiority trial. Lancet. 2012;379(9827):1721-7.

16. Piaggio G, Elbourne DR, Pocock SJ, Evans SJ, Altman DG, Group C. Reporting of noninferiority and equivalence randomized trials: extension of the CONSORT 2010 statement. JAMA. 2012;308(24):2594-604.

17. Koenker R, Machado JAF. Goodness of fit and related inference processes for quantile regression. J Am Stat Assoc. 1999;94(448):1296-310.

18. Nirmala K, Zainuddin AA, Ghani NA, Zulkifli S, Jamil MA. Carbetocin versus syntometrine in prevention of post-partum hemorrhage following vaginal delivery. J Obstet Gynaecol Res. 2009;35(1):48-54.

19. Boucher M, Nimrod CA, Tawagi GF, Meeker TA, Rennicks White RE, Varin J. Comparison of carbetocin and oxytocin for the prevention of postpartum hemorrhage following vaginal delivery: a double-blind randomized trial. J Obstet Gynaecol Can. 2004;26(5):481-8.

20. Carroli G, Cuesta C, Abalos E, Gulmezoglu AM. Epidemiology of postpartum haemorrhage: a systematic review. Best Pract Res Clin Obstet Gynaecol. 2008;22(6):999-1012

21. D'Agostino Sr RB, Massaro JM, Sullivan LM. Non-inferiority trials: design concepts and issues - the encounters of academic consultants in statistics. Stat Med. 2003;22(2):169-86.

22. FDA. Guidance for Industry, Non-Inferiority Clinical Trials: US Food and Drug Administration (FDA). 2010.

\section{Submit your next manuscript to BioMed Central and we will help you at every step:}

- We accept pre-submission inquiries

- Our selector tool helps you to find the most relevant journal

- We provide round the clock customer support

- Convenient online submission

- Thorough peer review

- Inclusion in PubMed and all major indexing services

- Maximum visibility for your research

Submit your manuscript at www.biomedcentral.com/submit 